\title{
La influencia de la orientación sexual de hombres y mujeres en las características acústicas de las vocales del español. Un estudio vocálico comparativo
}

\section{The Influence of Men And Women Sexual Orientation on the Acoustic Features of Spanish Vowels. A Comparative Vocalic Study}

\section{Jaime Crisosto A. \\ Fonoaudiólogo \\ Universidad de Concepción}

\section{Hernán Pérez $M$.}

Doctor en Lingüística

Universidad de Concepción

\section{Franco Dagnino R.}

Ingeniero Estadístico Universidad de Concepción
Contacto con el autor: Jaime Crisosto $A$. Universidad de Concepción, Facultad de Humanidades y Arte Edmundo Larenas $\mathrm{s} / \mathrm{n}$ Concepción - Chile Tel: (56 41) 2204404 Correo-e: jaimecrisosto@gmail.com

\begin{abstract}
RESUMEN
Históricamente se han asociado, desde posiciones más bien intuitivas, ciertas características de habla con la identidad de comunidades con una orientación sexual no heterosexual, espacio desde el cual también se configuran factores que emanan desde el binarismo de lo masculino y lo femenino. A partir de la década de 1990, la comunidad científica se ha empeñado en dilucidar cuáles son dichas características prosódicas, articulatorias, acústicas y lingüísticas que poseen los hablantes con una preferencia sexual más diversa. El propósito del artículo es determinar las características acústicas de las vocales del español de Chile (/i/, /e/, /a/, /o/, /u/) presentes en palabras aisladas, leídas en voz alta por hablantes heterosexuales y homosexuales de ambos sexos para establecer cuáles son las características que los identifican, organizando los datos obtenidos por orientación sexual. El método adoptado incluyó lectura en voz alta, aplicación de exclusiones, relación de datos y análisis estadístico de algunos de ellos. En términos generales, no existen marcadores acústicos vocálicos definidos que permitan diferenciar la orientación sexual de hombres y mujeres. Se requiere considerar otro tipo de información lingüística para poder establecer dichas diferencias, si es que las hubiera.
\end{abstract}

Palabras clave: orientación sexual, vocales, marcadores acústicos, fonética.

\begin{abstract}
Historically, from intuitive-oriented positions, certain features of speech have been associated with the identity of communities with a sexual orientation which is not heterosexual. In this respect, some factors are configured from the binary view of what is masculine and what is feminine. From the 1990s, the scientific community has tried to identify the prosodic, articulatory, acoustic and linguistic features which speakers with a different sexual preference have. The purpose of this study was to determine the acoustic characteristics of vowels of Chilean Spanish (/i/, /e/, /a/, /o/, /u/) in single words, read aloud by heterosexual and homosexual speakers, of both genders. The method adopted included reading aloud, data relation, exclusion criteria application and statistical analysis of some data. In general, there are no defined vocalic-acoustic markers to distinguish the sexual orientation of men and women. It is required to consider other linguistic information to establish differences.
\end{abstract}

Keywords: sexual orientation, vowels, acoustic markers, phonetics. 


\section{Introducción}

Desde hace algunas décadas, el estudio del habla homosexual ha comenzado a recibir cierta atención por parte de los círculos académicos. De tal modo, Darsey (1981) propone el denominado "teorema" que indica que "el hecho de que los gays hagan $X$, no hace a X gay". Más tarde, Kulick (2000), indica que cualquier investigación lingüística que estudie el fenómeno del habla homosexual precisa establecer que aquello por estudiar debe ser empírica y exclusivamente gay $\mathrm{y}$, al mismo tiempo, también debe aportar para definir qué es la homosexualidad. En tal panorama, este tipo de investigaciones puede adoptar dos rumbos distintos: tener como objeto de estudio el habla de personas que son abiertamente gays o lesbianas, o bien, realizar un estudio transversal para luego asignar categorías desde un punto de vista construccionista, explorando de qué manera ciertas prácticas lingüísticas materializan en el desempeño a los hablantes como gays o lesbianas mediante estudios perceptuales. Evidentemente, cada una de estas opciones encierra una serie de dificultades epistemológicas.

Una dificultad conceptual básica indicada por Kulick (2000), que no ha sido resuelta en este tipo de estudios, queda ejemplificada en el caso hipotético en el cual si los oyentes fuesen capaces de discriminar con un $100 \%$ de precisión a aquellos hablantes homosexuales de los que no lo son, aún no sabríamos qué exactamente es lo que está siendo identificado: ¿la orientación sexual como tal y, por ende, es aplicable a todos (¿la mayoría?, ¿algunos?) gays y lesbianas, e incluso a aquellos no asumidos? o bien, ¿es un estilo particular representacional en el cual, de alguna manera, la homosexualidad está indexada estereotípicamente, pero que solo ciertos gays y lesbianas utilizan (constituyendo, por ende, una decisión) y que, como consecuencia, nadie que quiera "transmitir homosexualidad" emplearía?

Dadas estas condiciones, Leap (1996) manifiesta que, según las implicancias del teorema de Darsey y la futilidad del ejercicio descriptivo, es necesario ampliar los márgenes desde la investigación en boga hasta finales de la década de los 90, ofreciendo un sistema teórico analítico-explicativo que permita otorgar coherencia al cuerpo de descubrimientos realizados en el área.

\section{Parámetros acústicos del habla homosexual versus habla heterosexual}

En torno a la frecuencia fundamental, el estereotipo de que los hombres homosexuales presentan un tono de voz más alto no ha demostrado ser verdadero en varias investigaciones que correlacionan ambas variables (Smyth, Jacobs y Rogers, 2003; Pierrehumbert, Bent, Munson, Bradlow y Bailey, 2004). Lo anterior no quiere decir, sin embargo, que los hombres homosexuales nunca usen un tono de voz (pitch) más elevado para expresar una identidad gay o femenina (Smyth y Rogers, 2008). En relación con la información acerca de las características acústicas del habla de las mujeres lesbianas, Moonwomon-Baid (1997) indica que el habla conversacional de las mujeres lesbianas y bisexuales presenta un rango de frecuencia fundamental más restringido que el de las mujeres heterosexuales. Por el contrario, Waksler (2001) no encuentra características acústicas que permitan determinar diferencias entre las mujeres 
heterosexuales y homosexuales. Es así que el tono, las variaciones prosódicas y la resonancia permiten establecer si la voz que escuchamos pertenece a un hombre o a una mujer. La calidad vocal, la duración de los segmentos y la articulación parecen ser información útil al determinar la masculinidad o femineidad de una voz (Davies y Goldberg, 2006).

Respecto de la articulación de las vocales, Pierrehumbert et al. (2004) afirman que tanto los hombres homosexuales como las mujeres lesbianas y bisexuales presentan un triángulo vocálico más abierto que los hablantes heterosexuales. Lo anterior contradice el supuesto de que las mujeres con una sexualidad distinta a la heterosexual presentan un comportamiento similar a un hombre heterosexual (Smyth y Rogers, 2008).

Por otra parte, en relación con la duración de los segmentos vocálicos, la revisión de Smyth y Rogers (2002) señala que son variados los estudios que demuestran una mayor duración de las vocales en mujeres que en hombres, en varias lenguas. Este hecho varía, sin embargo, dependiendo de si las vocales son tónicas o átonas, porque la diferencia significativa se observa en las primeras. Algo similar ocurre al comparar a hombres heterosexuales con hombres homosexuales, donde las vocales de estos últimos presentan mayor duración, pero solo en las más anteriores del triángulo. Sin embargo, si bien la investigación de Pierrehumbert et al. (2004) reporta vocales más largas para mujeres que para hombres, replicando lo que se había observado en estudios anteriores, no aparece una correlación significativa entre la duración y la orientación sexual del hablante.
Ahora bien, las diferencias anatómicas y fisiológicas en la conformación laríngea y del tracto vocal contribuyen de modo sustancial a las características acústicas de los sonidos, sin embargo, son muchos los aspectos de la estructura fonéticoacústica que no pueden ser explicados simplemente por estas diferencias reflejando, por lo tanto, procesos de aprendizaje social (Munson, 2011). Con todo, aún no está claro cuáles son las fuentes de este aprendizaje o los modelos mediante los cuales las personas con determinada identidad sexual funcionan lingüísticamente. Por otra parte, los rasgos de cada comunidad lingüística determinarán, en gran medida, cómo es que las características acústicas de una voz son categorizadas en una clasificación determinada. Algunas investigaciones sugieren que los oyentes realizan evaluaciones basadas en patrones fonético-acústicos estratificados, existiendo pistas socioindexadas que estos utilizan para evaluar la identidad social masculina/femenina (y probablemente, por extensión, la orientación sexual).

Cabe señalar que si bien la dicotomía tradicional de género "masculino" versus "femenino" o de orientación sexual "heterosexual" versus "homosexual" puede revelar algunas características importantemente diferenciadoras de cada género y/o sexo, las cuales resultan muy gruesas para intentar responder cuestiones específicas acerca de la identidad de género, la preferencia sexual y el habla. Por ejemplo, resulta lógico pensar que incluso dentro de la población de mujeres heterosexuales, algunas de ellas posean una voz con características acústicas más masculinas que otras y que este "índice de masculinidad" varíe, o no, en las clases 
más acomodadas o en diversas condiciones laborales (Smyth y Rogers, 2002).

La presente investigación pretende determinar cuáles son los parámetros acústicos que caracterizan el habla de hombres y mujeres heterosexuales y homosexuales mediante el análisis de las vocales del español de Chile.

\section{Método}

El propósito del estudio fue determinar las características acústicas de las vocales del español de Chile (/i/, /e/, /a/, /o/, /u/) presentes en palabras aisladas, leídas en voz alta por hablantes heterosexuales y homosexuales de ambos sexos, para establecer cuáles son las características que identifican a estos hablantes, organizando los datos obtenidos por orientación sexual.

\section{Participantes}

La cantidad de participantes del estudio fue de 20 hablantes nativos de español (cinco hombres heterosexuales, cinco hombres homosexuales, cinco mujeres heterosexuales y cinco mujeres homosexuales). Los participantes fueron reclutados por los investigadores, previo conocimiento de su orientación sexual. Cada uno de ellos debió asistir a la toma de muestras y completar una ficha de datos y el consentimiento informado, documentos de uso exclusivo de los investigadores para fines académicos. No se hizo referencia a la orientación sexual ni antes ni durante el experimento, para evitar la aparición de sesgos que pudieran afectar la validez de la muestra. Una vez que los datos fueron recolectados, se informó a los participantes acerca del objetivo del estudio y se les otorgó la posibilidad de retractarse de su participación en el proyecto y destruir el material grabado, pero ninguno mostró interés en dicha opción. Los procedimientos son concordantes con la Declaración de Helsinki (1975).

Todos los participantes poseían entre 20 y 28 años. No hubo diferencia estadísticamente significativa en la edad de hombres homosexuales y heterosexuales ( $p$ valor ANOVA 0.7). Tampoco se observó diferencia estadísticamente significativa en la edad de mujeres homosexuales y heterosexuales ( $p$ valor ANOVA 0.34).

Todos los participantes del estudio eran profesionales o estudiantes universitarios de la ciudad de Concepción. El test de Shapiro-Wilk de normalidad no evidencia que la distribución etaria entre hombres y mujeres de diferente orientación sexual difiera significativamente de la distribución normal. El test de Levene de homogeneidad, en tanto, muestra que la distribución etaria entre hombres y mujeres de diferente orientación sexual presenta homogeneidad de varianza entre ambos grupos. El test de Durbin-Watson, por último, arroja que los datos son independientes.

Los criterios de exclusión fueron: el uso de frenillos u otro dispositivo de ortodoncia, diagnóstico de trastorno auditivo, de lenguaje, habla o voz y/o estar actualmente en tratamiento fonoaudiológico. Una participante potencial del experimento manifestó haber sufrido de disfonía por nódulos vocales, frente a lo cual se decidió excluirla del estudio y buscar una nueva participante.

Todos los participantes provienen de la Región del Biobío (octava región) o de la Región de La Araucanía (novena región) de Chile, por lo que es 
esperable que utilicen un dialecto del español de Chile similar.

No se consideró un incentivo económico para la participación en el estudio.

\section{Estímulos}

Los estímulos utilizados en el estudio consistieron en 15 palabras monosilábicas. Las palabras seleccionadas evidencian la presencia de las cinco vocales del español (/i/, /e/, /a/, /o/, /u/) en un contexto consonante-vocal-consonante, sin presencia de consonantes nasales para minimizar los efectos de coarticulación: vil, tic, bis, res, tez, ser, ras, cal, tal, sol, tos, voz, tus, pus, luz.

\section{Procedimiento de grabación}

Durante el experimento se les presentaron a los participantes las palabras una a una en el centro de la pantalla de un notebook Toshiba Satellite C605, mediante software Microsoft Office PowerPoint 2007, con letras negras tamaño 72, fuente Calibri. Cada participante leyó la lista una sola vez para no condicionar la cantidad de palabras con variaciones prosódicas. No se entregaron instrucciones especiales antes de la grabación.

Los datos fueron grabados en un equipo digital TASCAM DR-100mkll en formato WAV con conexión a un micrófono dinámico unidireccional SHURE SM48, con una frecuencia de muestreo de 44100 y una cuantización de 24bit. El micrófono fue posicionado en un ángulo de $45^{\circ}$ respecto de la boca del hablante, a una distancia aproximada de $15 \mathrm{~cm}$ de la misma y la grabación se efectuó en una sala silenciosa.
Los datos acústicos fueron obtenidos utilizando el programa Praat (Boersma y Weenik, 2001). El inicio y el final de cada uno de los segmentos vocálicos fue determinado mediante un criterio visual a través de espectrograma de banda angosta (Window Length $=$ 0.03) para así determinar con mayor claridad la transición formántica. Todos los valores fueron obtenidos mediante las funcionalidades de Praat sin agregar ningún script. No fue necesario excluir ningún token vocálico debido a ruido externo, disfluencia del hablante o errores de lectura.

Las variables medidas fueron FO (medido en $\mathrm{Hz}$ ), F1 (medido en $\mathrm{Hz}$ ), F2 (medido en $\mathrm{Hz}$ ), F1 normalizado mediante procedimiento Nearey 1 (medido en unidades ad hoc), F2 normalizado mediante procedimiento Nearey 1 (medido en unidades ad hoc), Duración (medido en milisegundos), FO mínimo (medido en $\mathrm{Hz}$ ), FO máximo (medido en $\mathrm{Hz}$ ), Variación de FO (medido en $\mathrm{Hz}$ ), Intensidad (medido en $\mathrm{dB}$ ) y el valor obtenido a partir de la diferencia entre el valor de la amplitud del primer armónico y del segundo armónico o spectral tilt (medido en $\mathrm{dB}$ ). Esta última variable se incluyó como un indicador de la calidad vocal, puesto que está demostrado que voces más sopladas o con deficiencias en el cierre glótico presentan un primer armónico mayor en amplitud en comparación con el segundo, diferencia que se reduce en voces que no resultan sopladas (Hillenbrand y Houde, 1996). La variable ya había sido introducida en investigaciones previas para determinar algún grado de diferenciación en la comparación de población heterosexual frente a la homosexual (Munson, McDonald, DeBoe y White, 2006). 
Para el análisis estadístico de las variables se estableció el cumplimiento de supuestos de normalidad de residuos, homogeneidad de varianzas e independencia de errores mediante el test de Shapiro-Wilk, test de Levene y test de DurbinWatson correspondientemente. Se aplicó ANOVA para descartar interacción; cuando las varianzas de los factores no se hallaron homogéneas, se procedió a aplicar el test de Kruskal-Wallis para tal fin, donde se consideró $p>0.05$ para descartar interacción. Una vez llevado a cabo tal procedimiento, se aplicó el test Kruskal-Wallis nuevamente para comparar los niveles del factor de orientación sexual (homosexuales versus heterosexuales) en caso de ausencia de normalidad de residuos, o test $\mathrm{T}$ cuando no se cumplió el supuesto de homogeneidad de varianzas; se consideraron diferencias estadísticamente significativas con $p>0.05$.

\section{Resultados}

Hombres. En las vocales /a/, /e/ y /o/ no se observaron diferencias estadísticamente significativas. En la vocal /i/, la única variable con diferencias significativas entre los dos grupos fue la duración de este segmento vocálico (Kruskal-Wallis = 0.024). El boxplot correspondiente se muestra en la Figura 1, observándose que la duración de la vocal /i/ del grupo homosexual fue significativamente mayor en comparación con el grupo heterosexual.

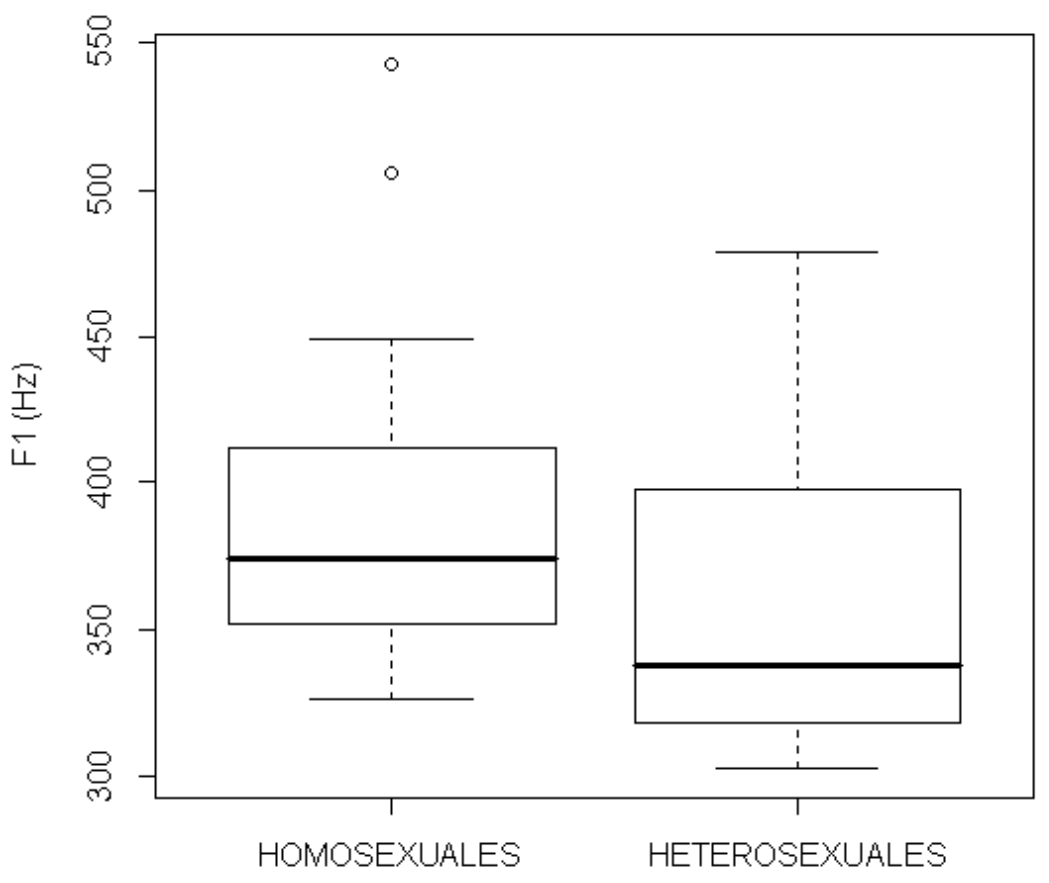

Figura 1. Boxplot duración vocal /i/ hombres homosexuales versus heterosexuales. 
En tanto, en la vocal /u/, la única variable que mostró divergencias significativas entre los dos grupos fue F1 (Kruskal-Wallis = 0.036). Sin embargo, cabe señalar que una vez que los datos vocálicos son normalizados, esta diferencia no se observa. El boxplot correspondiente se muestra en la Figura 2, evidenciando que $F 1$ de esta vocal del grupo homosexual fue significativamente mayor en comparación con la del grupo heterosexual. Se aprecia la presencia de dos valores atípicos en la muestra.

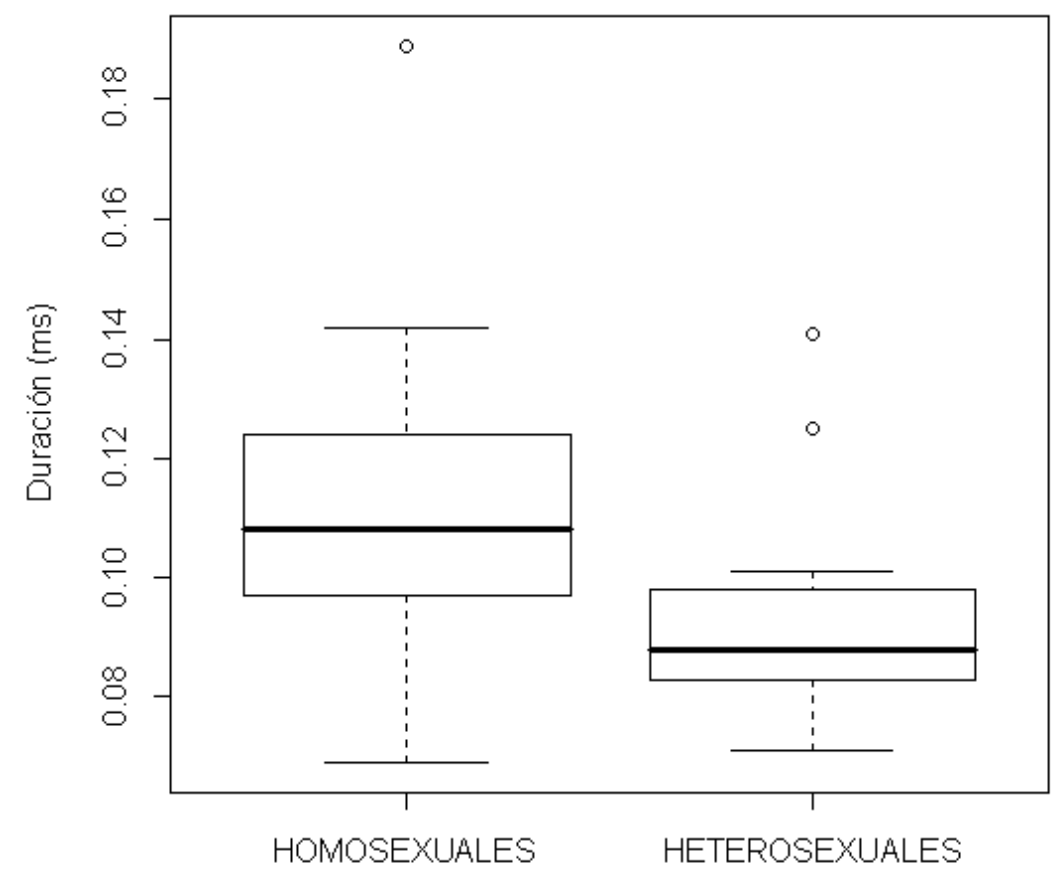

Figura 2. Boxplot F1 vocal /u/ hombres homosexuales versus heterosexuales.

Mujeres. En este caso, en las vocales /o/ y /u/ no se advirtieron diferencias estadísticamente significativas. En el caso de la vocal/i/, las únicas variables que mostraron diferencias significativas entre los dos grupos fueron F1 (ANOVA = $3.85 \mathrm{E}-0.5$ ), F1 Nearey 1 (Kruskal-Wallis $=0.00793$ ) y la pendiente espectral, Spectral Tilt (test T $=0.0056$ ).
La Figura 3 grafica el comportamiento de F1 de la vocal /i/ en la muestra. Se observa que F1 de las mujeres heterosexuales es significativamente mayor en comparación con las mujeres homosexuales.

La Figura 4 muestra que tal diferencia se mantiene incluso al someter a los valores de F1 al proceso de normalización mediante Nearey 1, observándose una distribución similar. 
Crisosto, J., Pérez, H., \& Dagnino, F.

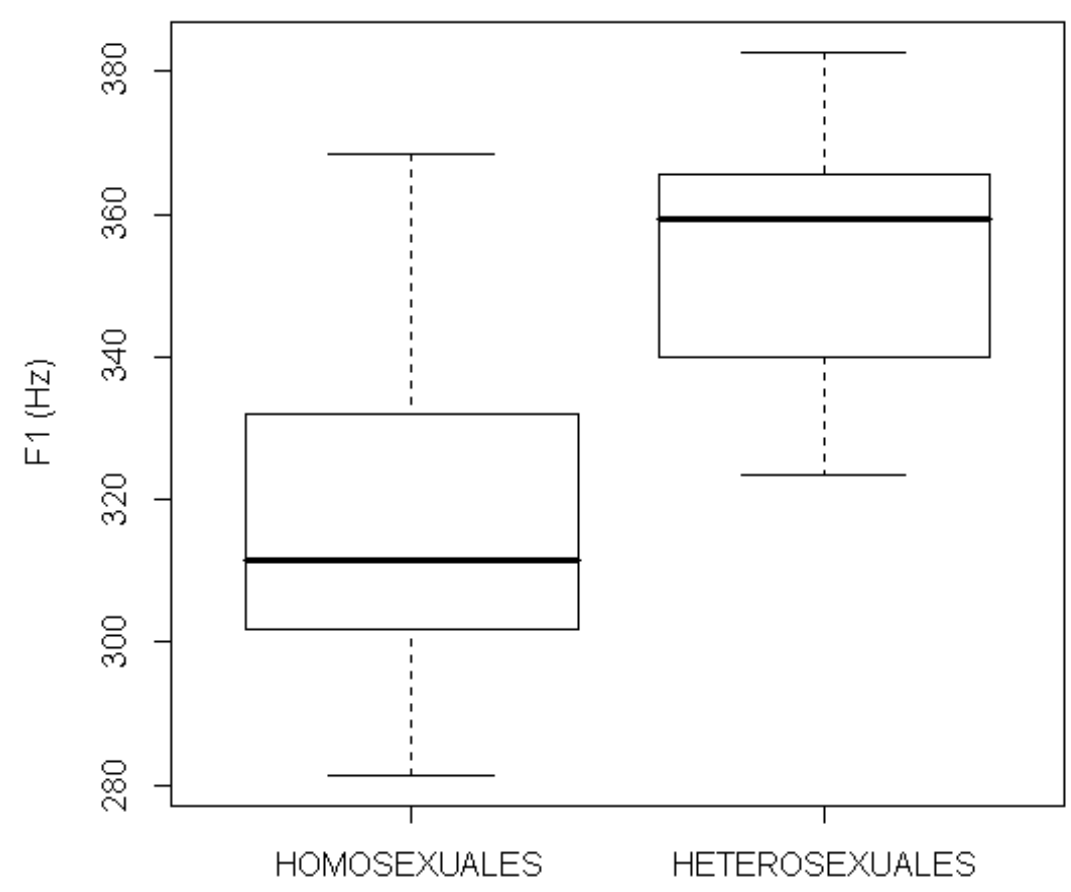

Figura 3. Boxplot F1 vocal /i/ hombres homosexuales versus heterosexuales.

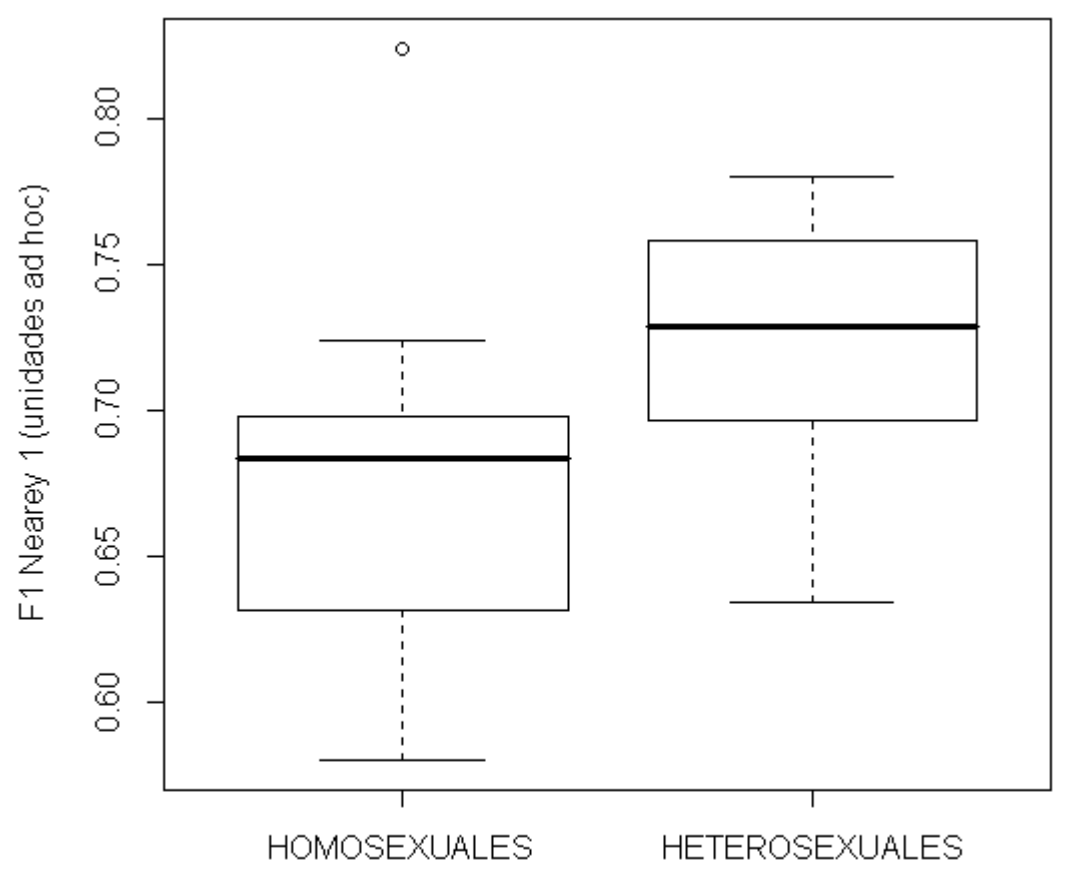

Figura 4. Boxplot F1 Nearey 1 vocal /i/ mujeres homosexuales versus heterosexuales. 
La Figura 5, en tanto, muestra que la pendiente espectral (Spectral Tilt) de la vocal/i/ de las mujeres heterosexuales es significativamente mayor en comparación con las mujeres homosexuales. La diferencia no se muestra tan evidenciable debido a la presencia del atípico en homosexuales, lo que reduce la escala y no permite la observación precisa de la diferencia.

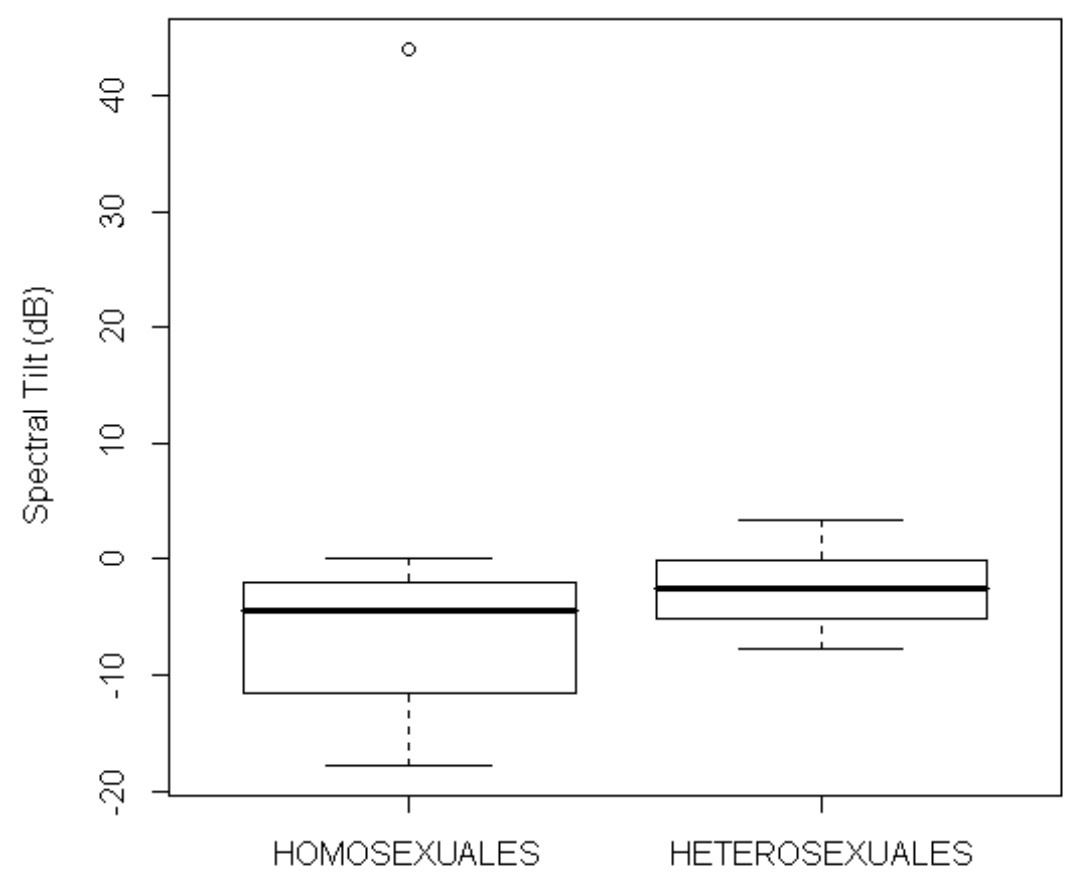

Figura 5. Boxplot F1 Nearey 1 vocal /i/ mujeres homosexuales versus heterosexuales.

Por otro lado, en la vocal /e/ las únicas variables que mostraron diferencias significativas entre los dos grupos fueron F0 (test $\mathrm{T}=0.0389$ ), FO mínimo (Kruskal-Wallis $=0.0191)$ y F0 máximo (Kruskal-Wallis $=0.0264)$.

La Figura 6 muestra que FO de la vocal /e/ de las mujeres homosexuales es significativamente mayor en comparación con las mujeres heterosexuales, aunque la dispersión de los datos en el primer grupo es mayor. Similar situación se observa en las Figuras 7 y 8 donde los valores de F0 mínimo y FO máximo, correspondientemente, son superiores en las mujeres homosexuales. 
Crisosto, J., Pérez, H., \& Dagnino, F.

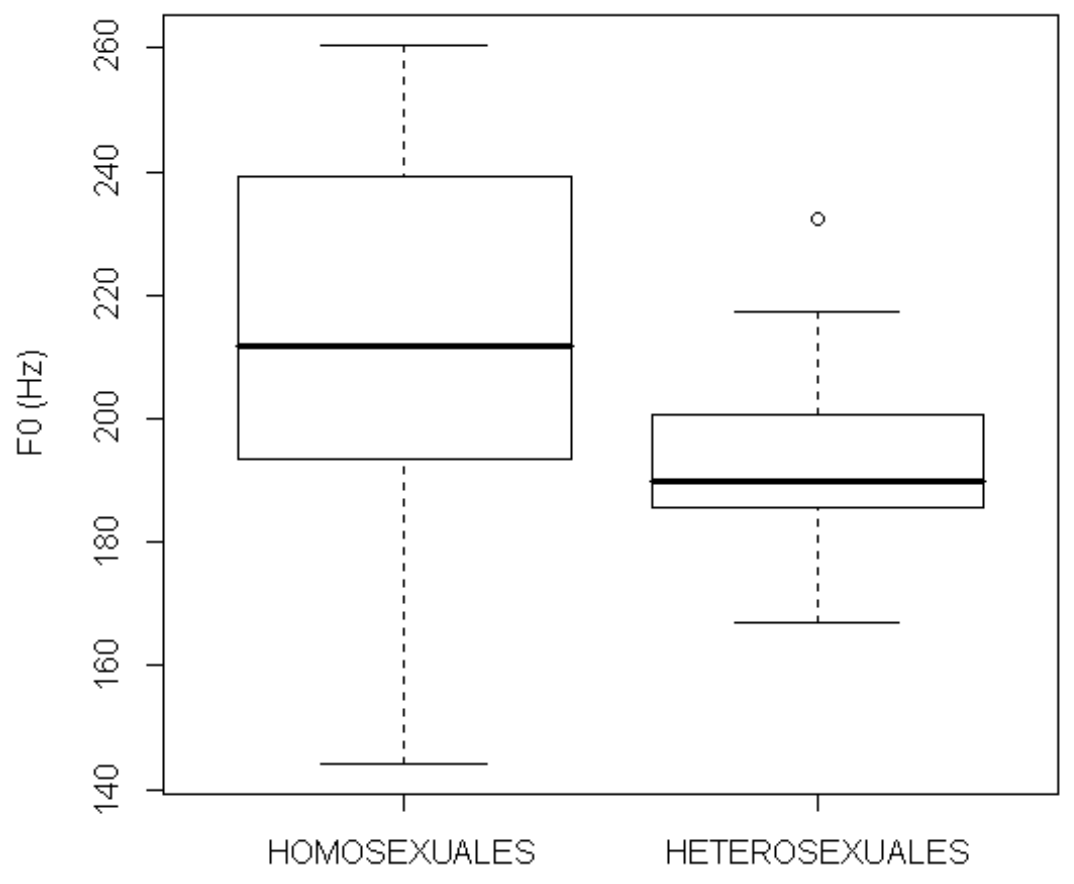

Figura 6. Boxplot F0 vocal /e/ mujeres homosexuales versus heterosexuales.

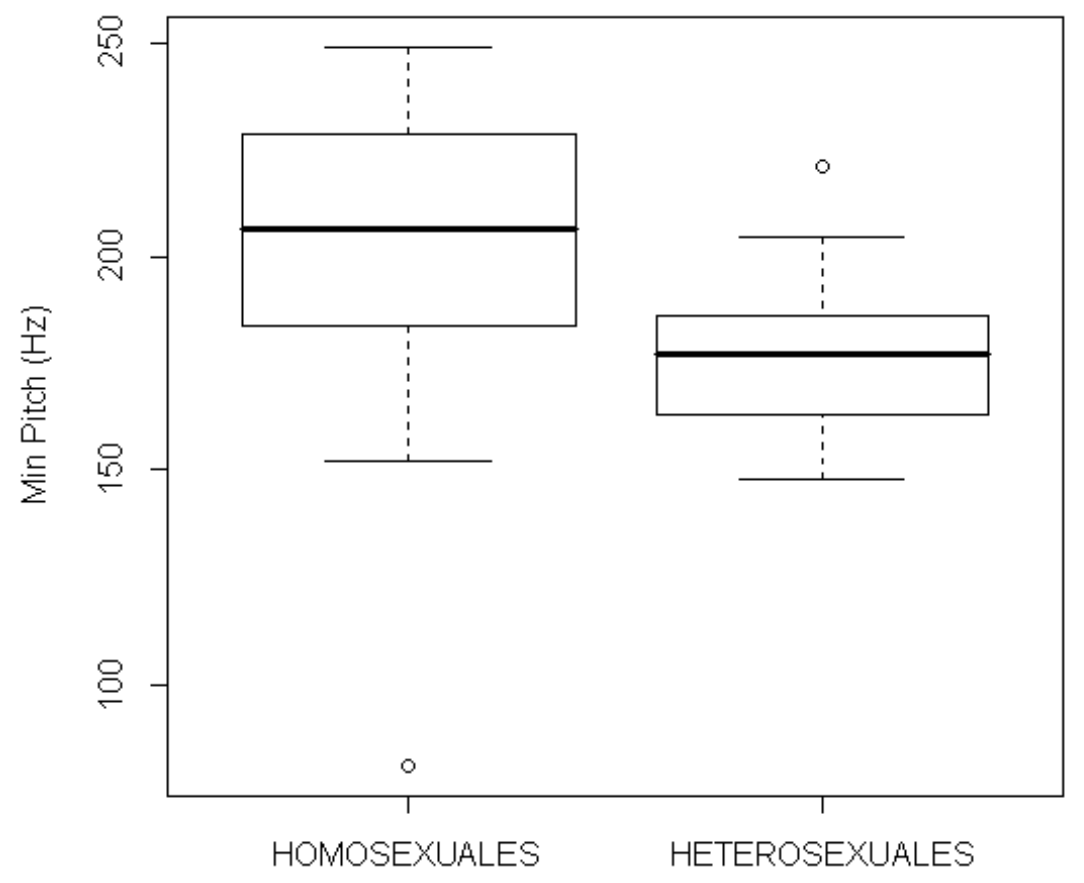

Figura 7. Boxplot F0 mínimo vocal /e/ mujeres homosexuales versus heterosexuales. 


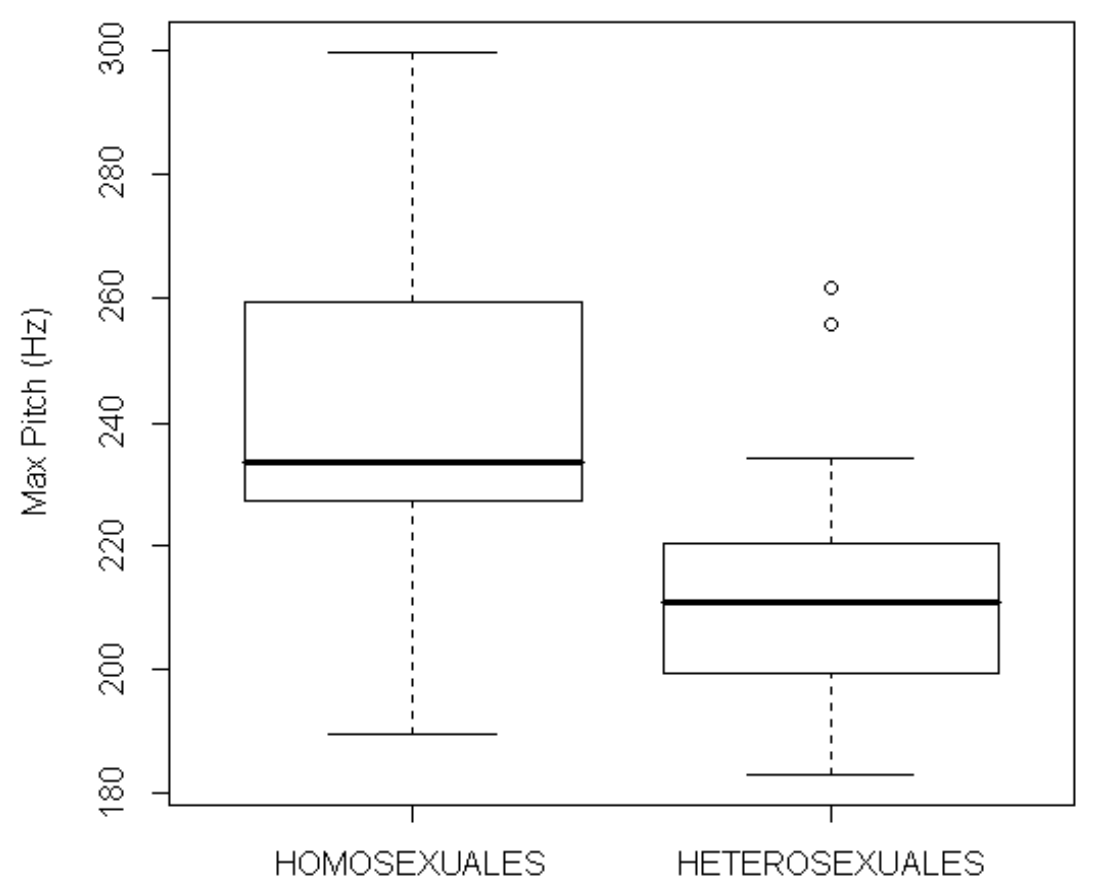

Figura 8. Boxplot F0 máximo vocal /e/ mujeres homosexuales versus heterosexuales.

En tanto, en la vocal /a/, la única variable que mostró diferencias significativas entre los dos grupos fue F0 máximo (Kruskal-Wallis $=0.0084$ ), siendo el F0 máximo de la vocal /a/ de las mujeres homosexuales significativamente mayor en comparación con las mujeres heterosexuales, tal como se muestra en la Figura 9.

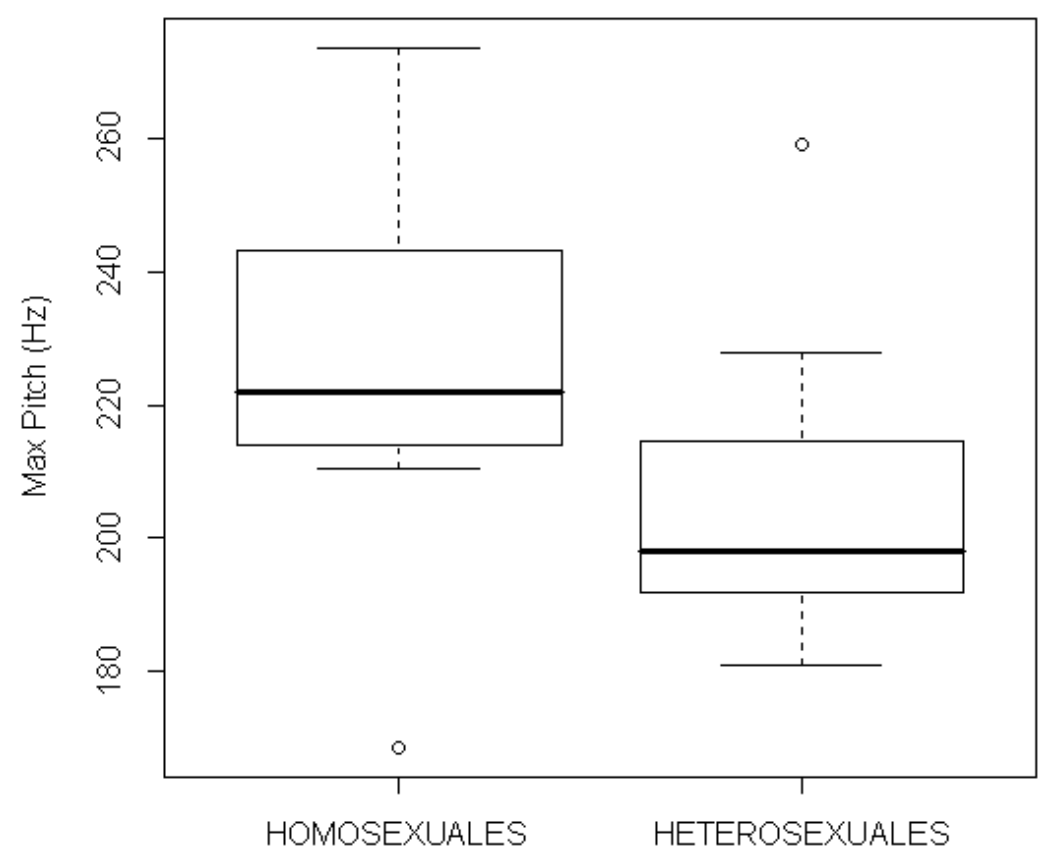

Figura 9. Boxplot F0 máximo vocal /a/ mujeres homosexuales versus heterosexuales. 


\section{Discusión}

Los resultados permiten afirmar en términos generales que no existen marcadores acústicos vocálicos definidos que permitan diferenciar la orientación sexual de hombres y mujeres, confirmando lo que indican investigaciones previas como las de Waksler (2001), Smyth y Rogers (2002) y Munson, McDonald, DeBoe y White (2006).

Tal como indican estas investigaciones, es posible que los hablantes homosexuales sí utilicen ciertas variaciones articulatorias o resonanciales que permitan transmitir su identidad sexual, pero esto no ocurriría en todos los contextos extralingüísticos. Llama la atención, además, que tal y como ocurre en estas investigaciones, F0 no demuestra ser un predictor adecuado de la orientación sexual de los hablantes, aun cuando intuitivamente se podría considerar como uno de los parámetros que podría presentar mayor correlación.

De este modo, las diferencias encontradas no siguen un patrón específico y parecen remitirse solo a ciertas vocales. Estas diferencias no parecen establecerse como un mecanismo claro de categorización lingüística, lo que supone que los sujetos hablantes no pueden ser catalogados en clases lingüísticas distintas considerando solo la información vocálica de manera aislada; podría ser necesario, entonces, considerar las curvas prosódicas, las características consonánticas o los indicadores de estabilidad de la señal acústica, como el jitter o shimmer para establecer dichas diferencias, si es que las hay.

Lo anterior también desecha la noción que indica que los homosexuales varones "hablan como mujeres" o que las mujeres lesbianas "hablan como hombres", punto discutido en algunas propuestas (Bailey, 2003) que avalan estas hipótesis para explicar algunas diferencias. Haciendo frente a esta cuestión, los sujetos parecen comportarse coherentemente con su sexo biológico y las diferencias halladas no responden a un acercamiento al sexo contrario.

Considerando la expansión de los triángulos vocálicos, tampoco es posible indicar que uno u otro grupo posea una articulación más "abierta" o "cerrada" que el otro. Los indicadores estadísticos exhiben que los hombres heterosexuales y homosexuales se comportan de manera similar y que lo mismo ocurre con las mujeres. Este hallazgo se contrapone a lo indicado por Pierrehumbert et al. (2004) quienes señalan que tanto hombres como mujeres homosexuales tendrían un espacio vocálico más amplio. Esta discrepancia podría explicarse a partir de dos diferencias metodológicas entre el presente estudio y el citado: primero, Pierrehumbert et al. (2004) llevan a cabo su experimento analizando las vocales en un contexto oracional, lo que lleva consigo marcas prosódicas que pueden influenciar las mediciones; y en segundo término, la lengua de análisis es el inglés, que posee un sistema vocálico distinto del español. Atendiendo a ambos aspectos, los resultados no se condicen con el escenario propuesto para la realización del presente estudio.

Por lo anterior no es posible realizar conclusiones acerca de variaciones articulatorias-resonanciales de cambios acerca del largo del tracto vocal, apertura mandibular o anteriorización y/o posteriorización lingual, por ejemplo, debido a que el comportamiento formántico no se observa de igual manera a lo largo de todo el triángulo vocálico; solo 
aparece cierta tendencia en relación con el comportamiento variable de F1 normalizado de /i/ en la comparación de mujeres heterosexuales versus homosexuales, lo cual establece una variable muy aislada e imposibilita poder entregar un panorama articulatorio que permita establecer aseveraciones más específicas acerca del comportamiento articulatorio vocálico de ambos grupos estudiados.

Para concluir, el estudio muestra discretas diferencias que no permiten afirmar la existencia de elementos acústicos distintivos en términos de la orientación sexual de hombres y mujeres siendo necesario, por ende, establecer otros niveles de análisis para complementar el estudio, o bien, determinar cuáles son los parámetros que operan en la mente de los individuos oyentes a la hora de juzgar una voz como heterosexual u homosexual.

\section{Referencias}

Boersma, P. y Weenink, D. (2001). Praat, a system for doing phonetics by computer. Glot International, 9-10(5), 341-345.

Bailey, J. M. (2003). The boy who would be queen: The science of gender-bending and transsexualism. Lincoln, NE: Joseph Henry Press.

Darsey, J. (1981). Gayspeak: A response. En J. Cheseboro (Ed.), Gayspeak: Gay male and lesbian communication (pp. 48-52). New York: The Pilgrim Press.

Davies, S. y Goldberg, J. (2006). Transgender speech feminization/masculinization: Suggested guidelines for BC clinicians. Recuperado de http://tsvoice.net84.net/media/guidelines-speech.pdf

Hillenbrand, J. y Houde, R. (1996). Acoustic correlates of breathy vocal quality. Dysphonic voices and continuous speech. Journal of Speech and Hearing Research, 39(2), 311-321. http://dx.doi.org/10.1044/jshr.3902.311
Kulick, D. (2000). Gay and lesbian language. Annual Review of Anthropology, 29(1), 243-285. http://dx.doi.org/10.1146/annurev.anthro.29.1.243

Leap, W. (1996). Studying gay English. En E. Lewin y W. Leap (Eds.), Out in the field: Reflections of lesbian and gay anthropologists (pp. 128-145). Illinois: University of Illinois Press.

Moonwomon-Baird, B. (1997). Toward a study of lesbian speech. En A. Livia y K. Hall (Eds.), Queerly phrased: Language, gender and sexuality (pp. 202-213). New York: Oxford University Press.

Munson, B., McDonald, E. C., DeBoe, N., y White, A. (2006). The acoustic and perceptual bases of judgments of women and men's sexual orientation from read speech. Journal of Phonetics, $34(2)$, 202-240. http://dx.doi.org/10.1016/j.wocn.2005.05.003

Munson, B. (2011). Lavender lessons learned: Or, what sexuality can teach us about phonetic variation. American Speech, 86(1), 14-31. http://dx.doi.org/10.1215/00031283$\underline{1277492}$

Pierrehumbert, J., Bent, T., Munson, B., Bradlow, A., y Bailey, J. M. (2004). The influence of sexual orientation on vowel production (L). The Journal of the Acoustical Society of America, 116(4), 1905-1908.

Smyth, R., Jacobs, G., y Rogers, H. (2003). Male voices and perceived sexual orientation: An experiment and theoretical approach. Language in Society, 32(3), 329-350. http://dx.doi.org/10.1017/s0047404503323024

Smyth, R. y Rogers, H. (2002) Phonetics, gender and sexual orientation. Recuperado de http://homes.chass.utoronto.ca/ claacl/2002/Smyth Rogers 2002.pdf

Smyth, R. y Rogers, H. (2008). Do gay-sounding men speak like women? Toronto Working Papers in Linguistics, 27, 129-144.

Waksler, R. (2001). Pitch range and women's sexual orientation. WORD, 52(1), 69-77. http://dx.doi.org/10.1080/00437956.2001.11432508 